\title{
Stability Analysis of Nonlinear Nuclear Reactor Cores Adopting T-S Fuzzy Modeling and Lyapunov Theory
}

\author{
Gang $\mathrm{Li}^{1,2,3,4,5}$, Bin Liang ${ }^{1,2,3,4,5, *}$, Xueqian Wang ${ }^{1,2,3,4,5}, \mathrm{Xiu}^{\mathrm{Li}}{ }^{1,2,3,4,5}$ and Kang Wang ${ }^{1,2,3,4,5}$ \\ ${ }^{1}$ Tsinghua University, National Laboratory for Information Science and Technology, 100084 Beijing, China \\ ${ }^{2}$ Tsinghua University, Graduate School at Shenzhen, 518055 Shenzhen, China \\ ${ }^{3}$ Tsinghua University, Department of Automation, 100084 Beijing, China \\ ${ }^{4}$ Shenzhen Key Lab of Space Robotic Technology and Telescience, 518055 Shenzhen, China \\ ${ }^{5}$ Shenzhen Engineering Lab of Precision Geometry Measurement Technology, 518055 Shenzhen, China \\ ${ }^{*}$ Corresponding author
}

\begin{abstract}
This research is to solve the stability analysis issue of nonlinear pressurized water reactor cores. On the basis of modeling a nonlinear pressurized water reactor core using the lumped parameter method, its linearized model is achieved via the small perturbation linearization way. Linearized models of the nonlinear core at six power levels are selected as local models of this core. The T-S fuzzy idea for the core is exploited to construct the T-S fuzzy model of the nonlinear core based on the local models and the triangle membership function, which approximates the nonlinear core model within the entire range of power level. This fuzzy model as a bridge is to cater to the stability analysis of the nonlinear core after defining its stability. One stability theorem is deduced to define the nonlinear core is globally asymptotically stable in the global range of power level. Finally, the simulation work and stability analyses are separately completed. Numerical simulations show that the fuzzy model can substitute the nonlinear core model; stability analyses verify the nonlinear core is globally asymptotically stable in the global range of power level.
\end{abstract}

Keywords-reactor core; T-S modeling; Lyapunov theory; stability analysis

\section{INTRODUCTION}

The precondition developing technologies of nuclear power plants (NPPs) for electricity generation is secure and stable operations of NPPs. Analyzing stability of objects in NPPs is always an important research direction promoted by community effort. A reactor core in a NPP is the key part as the hot source with radioactivity nuclear fuel, which possesses security risks and economic potential. Consequently, the study on stability of nuclear reactor cores is very necessary and meaningful. In the paper, the stability analysis of a nonlinear pressurized water reactor (PWR) core in a NPP is achieved modeling the core and adopting relative Lyapunov theories.

Since the PWR cores in NPPs are nonlinear and timevarying complex systems, modeling their models is difficult. Some researchers have worked on the modeling research on PWR cores. Of the work, the point reactor core modeling is popular. Kerlin et al. [1] modeled the PWR core of the H. B. Robinson NPP at the full power level which was also verified to be correct. PWR cores at the full power level were modeled by Edwards et al. [2-5] to design core power control systems. However, these modeling of cores at one power level cannot represent the dynamics of nonlinear core in the whole ranges of power level.

Generally, the analyzing methods for stability of nonlinear systems are to utilize Lyapunov theories to accomplish the stability analysis in the time domain and to adopt Popov theories to analyze the stability in the frequency domain [6]. The number of nonlinear systems that can be analyzed via the introduction of Popov theories is limit, and Lyapunov theories are feasible for the stability analysis of most nonlinear plants.

In this paper, the T-S fuzzy principle [7] of the nonlinear PWR core is exploited to model the T-S fuzzy model of the core on the basis of linearized local models of the core, which substitutes the nonlinear core model. Lyapunov theories and a deduced theorem based on the theories [8] [9] are used to analyze the stability of the core fuzzy model and the nonlinear core model by the linear matrix inequalities (LMI) solving method [10]. Finally, conclusions are provided.

\section{MODEL OF PWR CORE}

The point reactor core modeling as a traditional modeling method is adopted to model a PWR core, which is a lumped parameter method. For this modeling, a core is regarded as one point without any space profile, and parameters of the core only vary with time and have nothing to do with space positions.

According to the point reactor core modeling [1-3], the nonlinear PWR core is modeled adopting the point kinetics equations with six groups of delayed neutrons and reactivity feedbacks due to control rod movement and variations in fuel temperature and coolant temperature. Main model parameters are given in Table I. The nonlinear core model is showed as (1). 
TABLE I. MAIN MODEL PARAMETERS

\begin{tabular}{|c|c|}
\hline Parameter & Name \\
\hline$P_{r}$ & core power level \\
\hline$c_{r i}$ & $\begin{array}{c}\text { ith group normalized precursor } \\
\text { concentration }\end{array}$ \\
\hline$c_{r}$ & normalized precursor concentration \\
\hline$T_{f}$ & fuel average temperature \\
\hline$T_{i}$ & coolant inlet temperature \\
\hline$T_{e}$ & $\begin{array}{c}\text { coolant outlet temperature } \\
\text { position variation of the control rod } \\
\text { (fraction of core length) }\end{array}$ \\
\hline$\delta r o d$ & deviation of a parameter from initial steady \\
state value
\end{tabular}

$$
\left\{\begin{array}{l}
\frac{d P_{r}}{d t}=\frac{\rho-\beta}{\Lambda} P_{r}+\sum_{i=1}^{g} \frac{\beta_{i} c_{r i}}{\Lambda} \\
\frac{d c_{r i}}{d t}=\lambda_{i} P_{r}-\lambda_{i} c_{r i}, i=1,2, \ldots, g \\
\frac{d T_{f}}{d t}=\frac{f_{f} P_{0}}{\mu_{f}} P_{r}-\frac{\Omega}{\mu_{f}} T_{f}+\frac{\Omega}{2 \mu_{f}} T_{i}+\frac{\Omega}{2 \mu_{f}} T_{e} \\
\frac{d T_{e}}{d t}=\frac{\left(1-f_{f}\right) P_{0}}{\mu_{c}} P_{r}+\frac{\Omega}{\mu_{c}} T_{f}+\frac{2 M-\Omega}{2 \mu_{c}} T_{i}-\frac{2 M+\Omega}{2 \mu_{c}} T_{e} \\
\rho=\rho_{r o d}+\alpha_{f}\left(T_{f}-T_{f 0}\right)+\frac{\alpha_{c}}{2}\left(T_{i}-T_{i 0}\right)+\frac{\alpha_{c}}{2}\left(T_{e}-T_{e 0}\right) \\
\delta \rho_{r o d}=\alpha_{r o d} \delta r o d
\end{array}\right.
$$

One group delayed neutron model is utilized and the coolant inlet temperature is treated as a constant [3] [4].The small perturbation linearization methodology is utilized to linearize the nonlinear core model (1). The transfer function and the state equation of the core are separately calculated and expressed by

$$
\begin{gathered}
G=\frac{\delta P_{r}}{\delta \operatorname{rod}}=\frac{\sum_{i=0}^{3} a_{i} s^{i}}{\sum_{i=0}^{4} b_{i} s^{i}} . \\
\left\{\begin{array}{l}
\dot{\mathbf{x}}=A \mathbf{x}+B u \\
y=C \mathbf{x}+D u
\end{array}\right.
\end{gathered}
$$

where $\mathrm{u}=\delta$ rod-the input; $\mathrm{y}=\delta \operatorname{Pr}$-the output; ai $(\mathrm{i}=0,1,2,3)$ numerator coefficients; bi $(\mathrm{i}=0,1,2,3,4)$-denominator coefficients; s-laplace operator; $\mathrm{x}=[\mathrm{x} 1, \mathrm{x} 2, \mathrm{x} 3, \mathrm{x} 4] \mathrm{T}=[\delta \mathrm{Pr}, \delta \mathrm{cr}$, $\delta \mathrm{Tf}$, $\delta \mathrm{Te}] \mathrm{T}$-the state matrix; A-the $\mathrm{R} 4 \times 4$ system matrix; B-the $\mathrm{R} 4 \times 1$ input matrix; $\mathrm{C}$-the $\mathrm{R} 1 \times 4$ output matrix; $\mathrm{D}$-the zero matrix.

A transfer function of the core at a power level $10 * \mathrm{i} \%$ is denoted by $\mathrm{G} 10 * \mathrm{i} \%(\mathrm{i}=1,2,4,6,8,10)$. These transfer functions are calculated by using parameters from Ref. [11], in which the total primary heat output is $2200 \mathrm{MW}$, the primary coolant inlet temperature is $285 \mathrm{oC}$, the primary coolant outlet temperature is $317 \mathrm{oC}$, the primary coolant average pressure is 15.5 MPa, and the primary coolant mass flow is $12861.1 \mathrm{~kg} / \mathrm{s}$.

Let $\mathrm{G} 1=\mathrm{G} 10 \%, \quad \mathrm{G} 2=\mathrm{G} 20 \%, \quad \mathrm{G} 3=\mathrm{G} 40 \%, \quad \mathrm{G} 4=\mathrm{G} 60 \%$, $\mathrm{G} 5=\mathrm{G} 80 \%, \mathrm{G} 6=\mathrm{G} 100 \%$. The state equation of $\mathrm{Gi}$ is represented by ( $\mathrm{Ai} \mathrm{Bi} \mathrm{Ci} \mathrm{Di})(\mathrm{i}=1, \ldots 6)$. Parameters of $\mathrm{G} 6$ are enumerated: [a3, a2, a1, a0] $=\left[\begin{array}{l}50887021 \\ 1853137.2\end{array}\right]$, [b4, b3, $\mathrm{b} 2, \mathrm{~b} 1, \mathrm{~b} 0]=\left[\begin{array}{l}1 \\ 401.4\end{array} 692.2389 .9\right.$ 43.2]. G1, G2, G3, G4, G5 and G6 as local models of the nonlinear core model are used to respectively denote dynamics of the nonlinear core located in the six power level ranges $(0,10 \%],[10 \%, 20 \%],[20 \%, 40 \%]$, $[40 \%, 60 \%],[60 \%, 80 \%]$ and $[80 \%, 100 \%]$ in turn.

\section{T-S FUZZY MODELING FOR CORE}

The T-S fuzzy method [7] can be used to handle modeling issues of nonlinear plants such as the core at varying operating conditions. According to this modeling, the power level operating range of the nonlinear core is partitioned into separate subregions, local dynamics in each region are represented by one linear model as a local model of this core; fuzzy rules are proposed adopting membership functions that are utilized to develop fuzzy sets of which the intersection of two ones is nonempty, the T-S fuzzy model that is the overall model to approximate the nonlinear core is then achieved via "blending" of all linear local models based on fuzzy rules.

The triangle membership function is utilized to set up fuzzy rules. The membership function of the core fuzzy model at whole power levels is shown in Figure I.

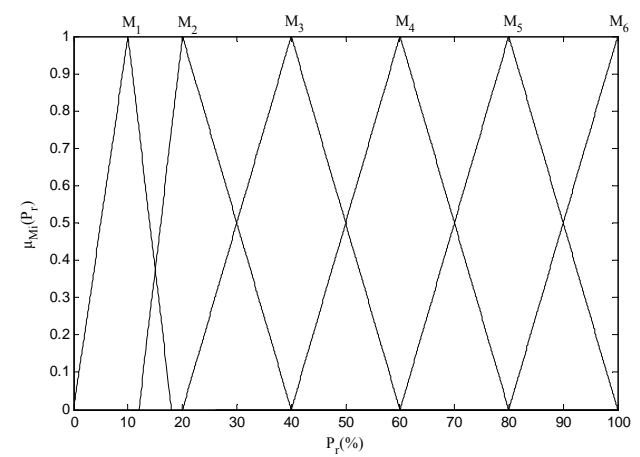

FIGURE I. MEMBERSHIP FUNCTION OF CORE FUZZY MODEL AT THE WHOLE POWER LEVELS

The fuzzy logic system (FLC) is of the following form.

Rule $\mathrm{i}$ : if $\operatorname{Pr}$ is $\mathrm{Mi}$, then $\mathrm{Yi}=\mathrm{Gi}(\mathrm{i}=1, \ldots 6)$

where Rule i denotes the ith fuzzy rule; M1, M2, M3, M4, M5 and M6 respectively represent the fuzzy sets corresponding to the power levels $10 \%, 20 \%, 40 \%, 60 \%, 80 \%$ and $100 \% ; \mu \mathrm{Mi}(\operatorname{Pr})$ represents the membership when a power 
Pr belongs to the fuzzy set Mi, which is calculated in the light of a relationship between the membership and the core power level.

In the light of Figure I, weight values qi for the "blending" of $\mathrm{Gi}(\mathrm{i}=1, \ldots, 6)$ is calculated as (4).

$$
q_{i}=\frac{\mu_{M_{i}}\left(P_{r}\right)}{\sum_{i=1}^{6} \mu_{M_{i}}\left(P_{r}\right)}, i=1, \ldots, 6
$$

Where $\operatorname{Pr} \in\left(\begin{array}{ll}0 & 1\end{array}\right], \mathrm{qi} \in\left[\begin{array}{ll}0 & 1\end{array}\right]$.

One value qi indicates how the ith local model at a power level belongs to a core fuzzy model at this power level. Finally, the core fuzzy model is expressed by (5) or (6).

$$
\begin{gathered}
G_{F M}=\sum_{i=1}^{6} q_{i} G_{i} \\
\left\{\begin{array}{l}
\dot{\boldsymbol{x}}=\sum_{i=1}^{6} q_{i}\left[A_{i} \boldsymbol{x}+B_{i} u\right] \\
y=\sum_{i=1}^{6} q_{i} C_{i} \boldsymbol{x}
\end{array}\right.
\end{gathered}
$$

\section{LYAPUNOV THEORY}

That reactor cores are stable is a guarantee of secure operations of NPPs. Hence, it is necessary to analyze the stability of the nonlinear PWR core. Theorem 1 is proposed to analyze the stability of this core. The key of the theorem is to solve common Lyapunov functions.

Based on (6), the dynamic part of the core fuzzy model GFM is expressed by (7).

$$
\dot{\boldsymbol{x}}=\sum_{i=1}^{6} q_{i} A_{i} \boldsymbol{x}
$$

According to the literature [8], Lemma 1 is shown to define the stability of the nonlinear core.

Lemma 1 Suppose the linear approximation GFM of the nonlinear core model is asymptotically stable, then the nonlinear core model is also asymptotically stable.

According to Lyapunov direct method [9], there are Lemma 2 and Lemma 3 shown as follows. Lemma 2 can be used to analyze the stability of the core fuzzy model GFM. In addition, Lemma 3 is the basis of deducing Theorem 1.
Lemma 2 Consider the linear core fuzzy model GFM $\dot{x}=\varphi(x)=\sum_{i=1}^{6} q_{i} A_{i} \boldsymbol{x}$ with $\varphi(0)=0$. Suppose that there exists a scalar function $V(x)$ such that $V(0)=0$; $V(\boldsymbol{x})>0$ and $\dot{V}(\boldsymbol{x})<0$ for $\boldsymbol{x} \neq 0 ; V(\boldsymbol{x}) \rightarrow \infty$ as $\|\boldsymbol{x}\| \rightarrow \infty$, then $V(x)$ is a Lyapunov function and the equilibrium state $(\boldsymbol{x})_{e}=0$ of the system $\dot{\boldsymbol{x}}=\varphi(\boldsymbol{x})$ is globally asymptotically stable.

Lemma 3 Consider one linear local model of the core fuzzy model described by $\dot{\boldsymbol{x}}=A_{i} \boldsymbol{x}$, then the equilibrium state $(\boldsymbol{x})_{e}=0$ is asymptotically stable in a large range on the sufficient and necessary condition that for a positive define real symmetric matrix $Q$ given discretionarily, there must be a positive define real symmetric matrix $\mathrm{N}$ such that $\mathrm{AiTN}+\mathrm{NAi}=-\mathrm{Q}$, namely $\mathrm{AiTN}+\mathrm{NAi}<0$.

Based on Lemmas 1, 2, 3, the stability theorem of the nonlinear core model is proposed as Theorem 1.

Theorem 1 The nonlinear core model is globally asymptotically stable on the sufficient condition that GFM is asymptotically stable, namely for all linear core local models expressed by $\dot{\boldsymbol{x}}=A_{i} \boldsymbol{x}$, there exists a common positive define real symmetric matrix $\mathrm{N}$, such that

$$
A_{i}^{\mathrm{T}} N+N A_{i}<0, i=1, \ldots, 6
$$

Proof:

Know that for the core fuzzy model (7), there is a positive define real symmetric matrix $\mathrm{N}$, such that $A_{i}^{\mathrm{T}} N+N A_{i}<0$. Take $V(\boldsymbol{x})=\boldsymbol{x}^{\mathrm{T}} N \boldsymbol{x}$ with $V(0)=0$ and $V(\boldsymbol{x}) \rightarrow \infty$ as $\|\boldsymbol{x}\| \rightarrow \infty$ as a common Lyapunov function for GFM, then for $\boldsymbol{x} \neq 0, V(\boldsymbol{x})$ is positive define and

$$
\dot{V}(\boldsymbol{x})=\sum_{i=1}^{6} q_{i} \boldsymbol{x}^{\mathrm{T}}\left(A_{i}^{\mathrm{T}} N+N A_{i}\right) \boldsymbol{x}<0
$$

According to Lemma 2, the core fuzzy model is globally asymptotically stable in the whole range of power level. Under the condition that GFM is the linear approximation of the nonlinear core model and globally asymptotically stable, the nonlinear core model is also asymptotically stable in a large range in the light of Lemma 1. Hence, the nonlinear core model is globally asymptotically stable in the whole range of power level. 


\section{STABILITY ANALYSIS}

In terms of Section IV, stability of the nonlinear core model is analyzed after verifying condition that GFM is the linear approximation of the nonlinear core model and globally asymptotically stable.
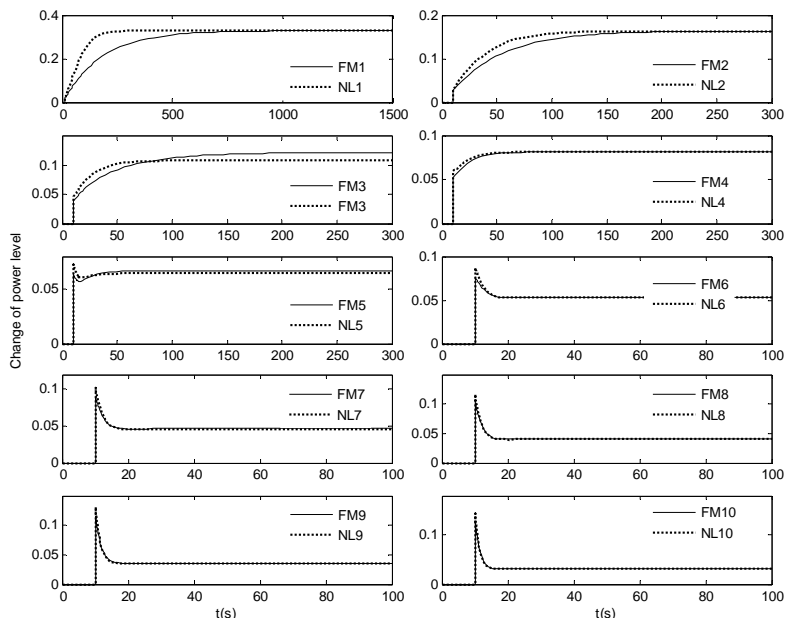

FIGURE II.

OUTPUT RESPONSES OF FUZZY MODEL AND NONLINEAR MODEL FOR THE CORE AT TEN POWER LEVELS

\section{A. Linear Approximation of The Nonlinear Core Model}

To verify that the GFM is the linear approximation of the nonlinear core model, the core fuzzy model (5) and the core nonlinear model (1) are compared via simulations.

When the input rod is taken as a 0.01 step, the fuzzy model and the nonlinear model at ten typical power levels $10 \times \mathrm{i} \%(\mathrm{i}=1, \ldots, 10)$ are simulated, and output responses of the fuzzy model and nonlinear model at these levels are shown in Figure II, which is namely responses of $\delta P r$. In Figure II, the curve FMi $(i=1, \ldots, 10)$ represents the output response of the fuzzy model at $10 \times \mathrm{i} \%$, and the curve NLi $(\mathrm{i}=1, \ldots, 10)$ is the output response of the nonlinear model at $10 \times \mathbf{i} \%$. From FMi and $\mathrm{NLi}$, it can be observed that FMi approaches $\mathrm{NLi}$. Similarly, the similar simulation results are able to be achieved for other power levels.

Therefore, the core fuzzy model can approximate the core nonlinear model according to simulation results, namely GFM is the linear approximation of the nonlinear core model.

\section{B. Stability of GFM}

The common positive define real symmetric matrix $\mathrm{N}$ is solved by programming based on the LMI toolbox [10] and (9). Each eigenvalue of $\mathrm{N}$ is a positive number and given by $\{1.25785 .1401785 .87608 .1626 \times 104\}$. Consequently, there is the common positive define real symmetric matrix $\mathrm{N}$ meeting Theorem 1 .

In terms of Theorem 1, the nonlinear core model is globally asymptotically stable in the global range of power level.

\section{ACKNOWLEDGMENT}

The authors would like to thank anonymous reviewers for their valuable comments. The work is funded by National High Technology Research and Development Program of China (863 Program) (No.2015AAXX46611) and China Postdoctoral Science Foundation (No.20159200078).

\section{REFERENCES}

[1] T.W. Kerlin, E.M. Katz, J.G. Thakkar, J.E. Strange, "Theoretical and experimental dynamic analysis of the H. B. Robinson nuclear plant," Nuclear Technology, vol. 30, pp. 299-316, 1976.

[2] R.M. Edwards, K.Y. Lee, M.A. Schultz, "State feedback assisted classical control: an incremental approach to control modernization of existing and future nuclear reactors and power plants," Nuclear Technology, vol. 92, pp. 167-185, 1990.

[3] A. Ben-Abdennour, R.M. Edwards, K.Y. Lee, "LQG/LTR robust control of nuclear reactors with improved temperature performance," IEEE Trans. on Nuclear Science, vol. 39, pp. 2286-2294, 1992.

[4] H. Arab-Alibeik, S. Setayeshi, "Improved temperature control of a PWR nuclear reactor using an LQG/LTR based controller," IEEE Trans. on Nuclear Science, vol. 50, pp. 211-218, 2003.

[5] G.R. Ansarifar, H.R. Akhavan, "Sliding mode control design for a PWR nuclear reactor using sliding mode observer during load following operation," Annals of Nuclear Energy, vol.75, pp. 611-619, 2015.

[6] L.Z Fu, Dynamics of Nuclear Reactor, Beijing: Atomic Energy Press, 1988, pp. 100-115.

[7] K. Tanaka, M. Sugeno, "Stability analysis and design of fuzzy control systems," Fuzzy Sets and Systems, vol. 45, pp. 135-156, 1992.

[8] A. Isidori, Nonlinear Control Systems, 3rd ed. New York: Springer, 1995, pp.137-178.

[9] B. Liu, W.S. Tang, Modern Control Theory, Beijing: China Machine Press, 2007, pp. 157-177

[10] A. Gahinet, et al., LMI Control Toolbox User's Guide, Natick: The MathWorks, Inc., 1995.

[11] IAEA, Directory of Nuclear Reactors Vol.IX, International Atomic Energy Agency, 1971, pp. 27-34. 